\title{
AXIOMATIC DESIGN USING ONTOLOGY MODELING FOR INTEROPERABILITY IN SMALL AGRICULTURE MACHINERY PRODUCT DEVELOPMENT
}

\author{
Jiandong Jiang, Fang Xu, Xinrong Zhen, Xian Zhang,Yangyu Wang, Libin \\ Zhang* \\ The MOE Key Laboratory of Mechanical manufacture and Automation, Zhejiang University \\ of Technology, Hangzhou, China, 31003.Email:jiangjd@zjut.edu.cn
}

Abstract: Small Agricultural machinery (SAM) is applied widely in agriculture, forestry and stockbreeding. We focus the reconfiguration and Interoperability of the complex process of SAM product development, and propose a design architecture employing axiomatic design method and ontology information representation. In the proposed design process, product function decomposing and recomposing according to independent axiom of axiomatic design, all information of all design phases is expressed in ontology information model with XML language so that can be communicated among all engineers. A case study conducted in Small Agriculture Machinery (SAM) design helps to illustrate the proposed approach, it shows the proposed methods are feasible and reliable, lays a foundation of SAM product reconfigurable design system.

Key words: Small agricultural machinery, Axiomatic design, Ontology, Gearbox

\section{INTRODUCTION}

Small Agricultural machinery (SAM) is applied widely in agriculture, forestry and stockbreeding. With the ever drastically increment of market competition and the increase of requirement for product personality, Companies must solve numerous problems that have different origins. First of all, products are increasingly complex and should answer a great number of requirements imposed by customers, and manufactures have to improve quality of their products, to have more creativity than their competitors and to reduce costs and times of production, and the key to solve above-

*This project is supported by the Natural Science Foundation of China (Grant 50375146), National Basic Research Program of China (Grant 2004CCA05800), The Doctoral Program Foundation of Ministry of Education of China (Grant 20040337002), the department of international Science\&Technology cooperation of China (Grant 2005DFA70380), Zhejiang Provincial department of international Science\&Technology cooperation of China (Grant 2005C14005) and Zhejiang Provincial Natural Science Foundation of China (Grant Z503054).

Please use the following format when citing this chapter:

Jiang, Jiandong, Xu, Fang, Zhen, Xinrong, Zhang, Xian, Wang, Yangyu, Zhang, Libin, 2006, in International Federation for Information Processing (IFIP), Volume 207, Knowledge Enterprise: Intelligent Strategies In Product Design, Manufacturing, and Management, eds. K. Wang, Kovacs G., Wozny M., Fang M., (Boston: Springer), pp. 184-191. 
mentioned problems lies in the product development which exhausts about $70 \%$ technical investment of enterprise. The difficulty to implement product development process can be explained by high number of different phases, and thus disciplines, that have to collaborate. Among all these phases, we can find (Ghodous, 1996, Prasad, 1996, Sriram, et al., 2002, Ullman, 1992, etc.): study of purposes, preliminary studies, planning, preliminary design, functional decomposition, detailed design of product, destruction and recycling. Due to all the phases (different objectives, constraints, manipulated information between functional design and manufacture, etc.) are difficult for them to communicate information.

We are interested in conceptual and virtual aspects of product development. Indeed, our objective is to find methods and data-processing tools, which can help experts to work in an individual and implement an automation design for product development. In this paper, we are involved in improvement of data communication from the first product development phases towards the most advanced one. More precisely, we focus on functional design and detailed design in small agricultural machinery (SAM) product development, and propose a method of axiomatic design using ontology modeling for Interoperability, as they are essential in the SAM product development process:

(1) The first one determines the main functions that are associated to an artifact and are mapped from customers requirements;

(2) The second one decomposes and recomposes these functions to generated the exact structural modal of the artifact employing axiomatic design theory and reconfigurable design methods;

(3) The last one builds a general representation model of design knowledge, experience and geometry information for all phases of product development.

These phases are difficult to relate because they involve different product artifact representations, Hence, our global objective is to find an artifact representation that would be common to these phases. To realize that, we decompose our work in two separate parts: we first consider form to physical structure mapping, which means that we try to find a way to improve interoperability between both functional design and detailed design and detailed design. The second part of our work consists to join the works that have been realized separately into a unique global approach for customer requirements-to-function mapping.

\section{AXIOMATIC DESIGN}

Axiomatic design provides a systematic and logical methodology for 
deriving, documenting and optimizing design processes. In the words of Professor Nam Suh of MIT, where the axiomatic design process was developed, axiomatic design methods work to make design more creative, reduce the random solution search process, minimize iterative trial-anderror processes and determine the best design among those proposed [Nam Pyo Suh, etc, 2001]. There are two axioms: (1) independence axiom; (2) information axiom.

Axiomatic design is a process in which engineers, designers and managers first think functionally and then follow through by the innovative creation of physical embodiment. This process provides a systematic way to satisfy many functional requirements (FRs) without introducing coupling of functions and creating integrated physical systems. It decomposes higherlevel FRs and design parameters (DPs) into leaf-level FRs and DPs that can be implemented to construct the system according to the resulting design decision architecture. Figure 1 shows an axiomatic process schematically.

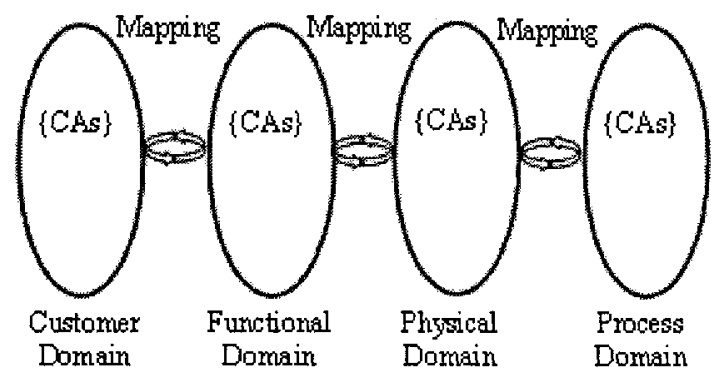

Functional requirements (FRs) are a minimum set of independent requirements that completely characterize the functional needs of the design solution in the functional domain. Design parameters (DPs) are the elements of the design solution in the physical domain that are chosen to satisfy the specified FRs.

Constraints (Cs) are bounds on acceptable solutions.

Process variables $(\mathrm{PV})$ are the elements of the process domain that characterize the process that satisfies the specified DPS.

Figure 1. Fundamental concept of axiomatic design.

\section{ONTOLOGY-BASED F-B-S INFORMATION MODEL}

In the process of product design, redesign namely modified design is applied agilely, and reuse of information and knowledge becomes the key to advance design quality and efficiency. So we focus on how to establish design information model to organize and reuse corresponding design information and knowledge at the circumstance of collaborative design. The 
conception of ontology (Natalya; Michael; Liu, 2002; Li, 2002) in artificial intelligence is: it is a group of concepts or nomenclatures. It researches on classification and attributions of objects and the relationships among objects.

We focus on how to establish a design information model which organizes and reuses design information and knowledge obtained from collaborative design. This paper proposes an ontology-driven design information model and applies it to SAM to realize common use of information and knowledge in product development phases. On account of the three principles (uniqueness, completeness and minimum) constituted by Bogoni at three aspects of product description including function, behavior and structure, a information model satisfying different principles respectively is proposed, and the ontological information model presents all information in the process of design, simulation and production.

\section{CASE STUDY}

There is a case study conducted in SAM design which illustrates the XML frame of the ontological information model. The description of XML frame for F-B-S model is as following:

Table 1. Ontology representation for speed reducer of SAM

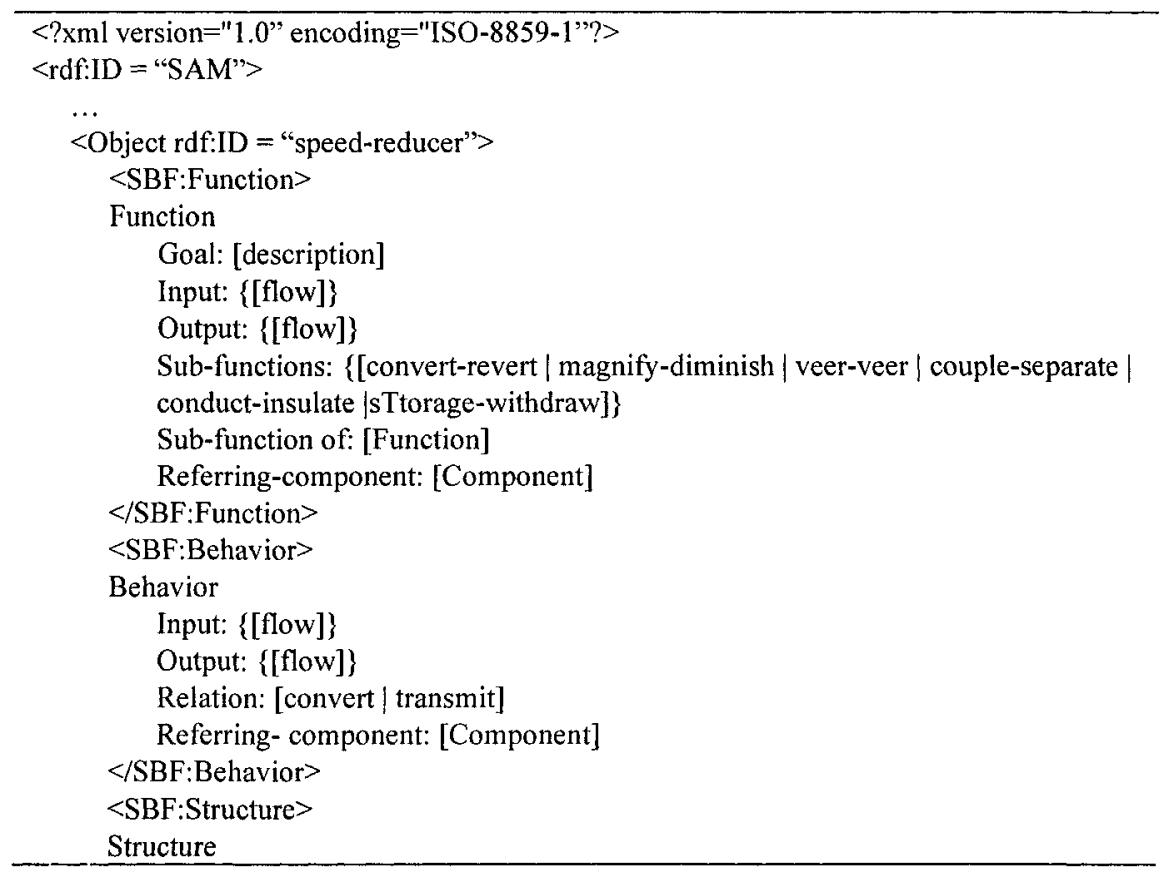




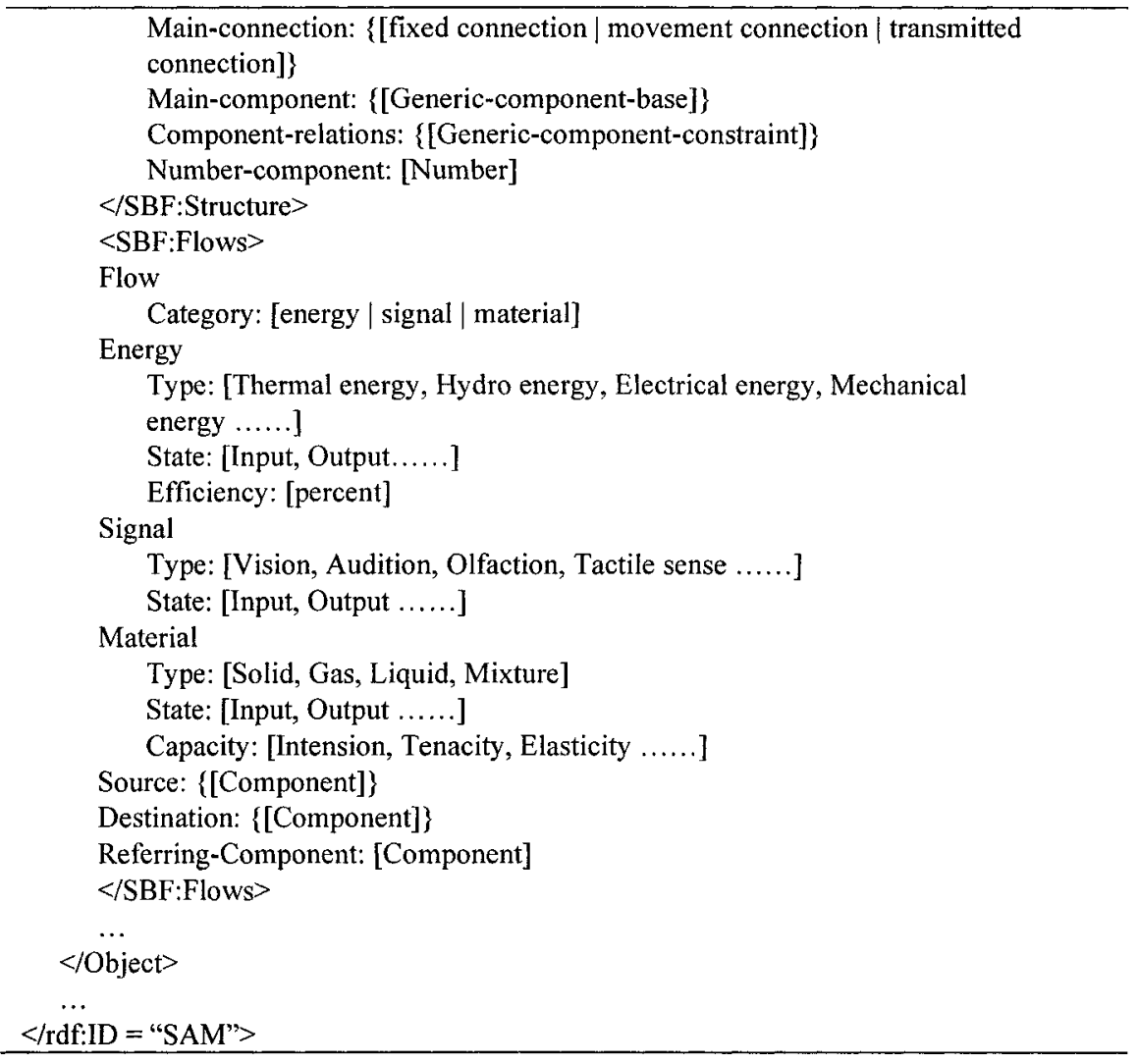

\section{ARCHITECTURE OF PRODUCT DESIGN SYSTEM}

Figure 2 illustrate the architecture of SAM product design system. It shows the SAM product design process according to four steps: the first transforms customer requirements into product functions employing product experts and sales through CRM tools and market investigation (Fig.2-A), the farther contents are referenced in our previous paper [Jiang Jiandong, et al., 2005 ,]. The second specifies how to decompose product functions according to independence axiom of axiomatic design method and how to recompose functions on principle of product module reconfiguration warranted by product library (Fig.2-B, C), detailed method is referred in interrelated literatures [Zhang Libin Jiang Jiandong, 2004, Zhang Libin, et al., 2005]. The third shows that a part, component and whole product will be expressed with functional ontology, behavioral ontology and structural ontology in individual phase, then a finished design is expressed in general ontology information model so that can be utilized by all engineers (Fig.2-D, E), it is 
referred in some literatures [Jiang jiandong, et al., 2005]. The last is that the purpose of designer is computed digitally, and translated into digital model in computer (Fig.2-F, G).

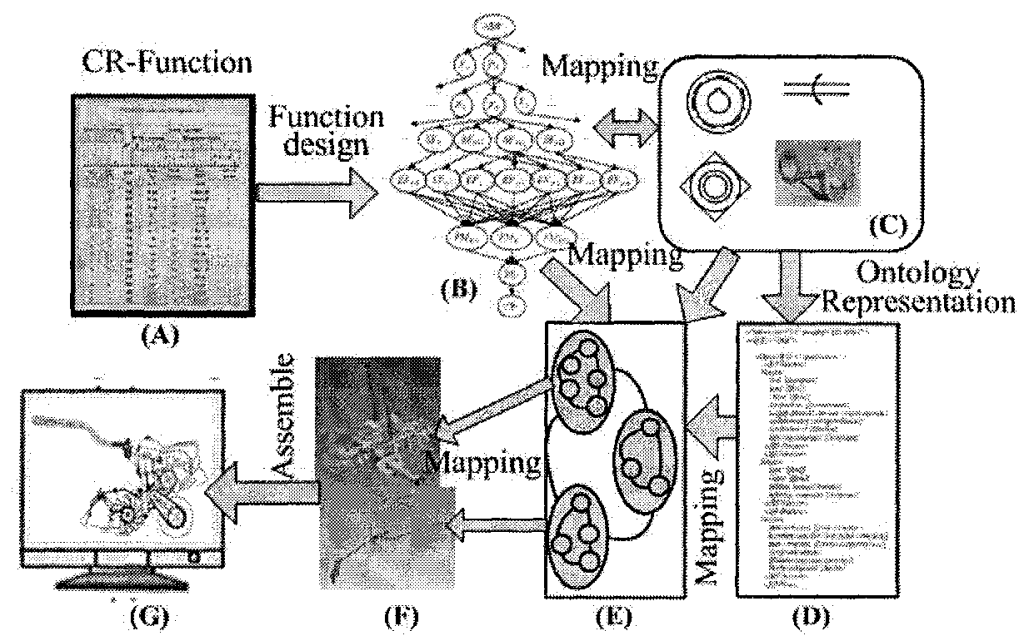

Figure 2. Design process of SAM product

The frame of product design system employs axiomatic design method, CRM, F-B-S ontology modeling reconfiguration design method and reconfigurable methods, integrates conceptual design, detailed design, intelligent design, artifact simulation and 3D product design, etc..

\section{APPLICATION OF AXIOMATIC DESIGN FOR SAM GEARBOX}

Axiomatic design theory adopts top-down decomposed method and designs function from abstract objects to detailed structure. A case study of SF series gearbox conducted in Small Agriculture Machinery (SAM) design helps to illustrate the proposed product function design and interrelated method. Firstly, the essential requirements include: low cost, small volume, agile manipulation and good adaptability, the mapped functions FRs and design parameters DPs may sort out: (1) $\mathrm{FR}_{1}$-various speed, $\mathrm{DP}_{1}$-gear design; (2) $\mathrm{FR}_{2}$-low cost; $\mathrm{DP}_{2}$-manufacture plan; (3) $\mathrm{FR}_{3}$-integrated gear and caseframe, $\mathrm{DP}_{3}$-case structural design; So the prime design matrix is shown in following 


$$
\left\{\begin{array}{l}
F R_{1} \\
F R_{2} \\
F R_{3}
\end{array}\right\}=\left[\begin{array}{ccc}
X & 0 & 0 \\
0 & X & 0 \\
X & X & X
\end{array}\right]\left\{\begin{array}{c}
D P_{1} \\
D P_{2} \\
D P_{3}
\end{array}\right\}
$$

According to SAM task environmental requirements, we decide four various speed gearbox including four-speed, three-speed, two-speed and one-speed. So the design matrix for $\mathrm{FR}_{1}$ shows:

$$
\left\{\begin{array}{l}
F R_{11} \\
F R_{12} \\
F R_{13} \\
F R_{14}
\end{array}\right\}=\left[\begin{array}{cccc}
X & 0 & 0 & 0 \\
0 & X & 0 & 0 \\
0 & 0 & X & 0 \\
0 & 0 & 0 & X
\end{array}\right]\left\{\begin{array}{l}
D P_{11} \\
D P_{12} \\
D P_{13} \\
D P_{14}
\end{array}\right\}
$$

Where, $F R_{11}$ is four-speed gearbox structural design, $F_{12}$ is three-speed gearbox structural design, $\mathrm{FR}_{13}$ is two-speed gearbox structural design, $\mathrm{FR}_{14}$ is one-speed gearbox structural design, $\mathrm{DP}_{1 i}(i=1 \sim 4)$ are design parameters for detailed structure of individual gearboxes.

All FRs and DPs are need decomposing and composing abided by independence axiom and information axiom, the final result includes almost eighty independent functional and one hundred design parameters, and these design parameters can be integrated into eighteen modules of SAM gearbox determined by forty-six structural parameters. The result is shown in Figure 3.

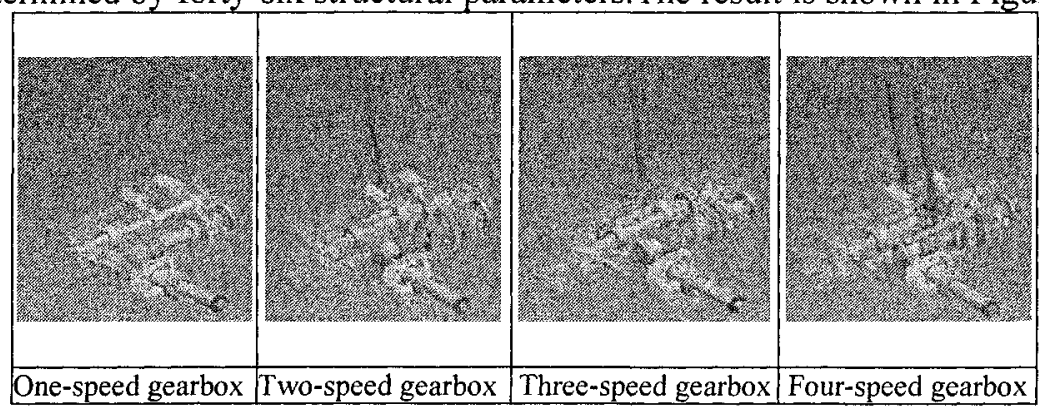

Figure 3. Artifacts for proposed design method

\section{CONCLUSION}

A design architecture employing axiomatic design method and ontology information representation is proposed. In the proposed design process, 
product function decomposing and recomposing according to independent axiom of axiomatic design, all information of all design phases is expressed in ontology information model with XML language so that can be communicated among all engineers. A case study of SF series gearbox is realized by axiomatic design method, and the functional, Behavioral and structural information for all design phases is expressed in ontology. This paper proves the feasibility and reliability by functions decomposed and mapping by actual SAM components. The proposed methods and design processes lay a foundation of SAM product reconfigurable design system.

\section{REFERENCE}

Ghodous, P. (1996), "Modélisation intégrée de données de Produit et de Processus de Conception", "Informatique", Lyon, Université Claude Bernard-Lyon 1.

Jiang Jiandong, Zhang Libin, Xu Fang, Zhang Xian, Ji Shiming, Lv Fangya. (2005), Study on the model of customer requirements for SAM product under MC. Nongye Gongcheng Xuebao/Transactions of the Chinese Society of Agricultural Engineering, v 21, $\mathrm{n}$ 9, September, p 98-102.

Jiang Jiandong, Zhang Libin, Xu Fang, Zhang Xian, Ji Shiming, Lv Fangya.(2004), Study on the model of customer requirements for SAM product under MC, Soypsium of national cross-strait manufacture technology, 15, Nov. p.80-85.

Liu Xijuan, Jiang Shouwei (2002), "Research on Reconfigurable Product Design Theory and Several Key Problems," thesis for doctor's degree, Shanghai Jiao Tong University, Shanghai, China. (In Chinese)

Li Shanping, Yin Qiwei, Hu Yujie (2004), "Overview of Researches on Ontology," Journal of Computer Research and Development, Vol. 41, No. 7, pp. 1041-1052. (In Chinese)

Michael Erdmann, Rudi Studer, "Ontologies as Conceptual Models for XML Documents," Institut für Angewandte Informatik und Formale Beschreibung sverfahren (AIFB) University of Karlsruhe (TH), D-76128 Karlsruhe (Germany).

Ullman, D. G. (1997), The mechanical design process, $2^{\text {nd }}$ edition, McGraw-Hill.

Nam Pyo Suh, et al (2001) Axiomatic design-advances and applications, Oxford University Press, Inc.

Natalya F. Noy, Deborah L. McGuinness, "Ontology Development 101: A Guide to Creating Your First Ontology, " Stanford University, Stanford, CA.

Prasad, B. (1996), Concurrent Engineering Fondamentals, 2, 478.

Sriram, R. D. (2002), Distributed and integrated collaborative engineering design, 1, Sarven Publishers.

Zhang Libin, et al (2005) "Scheme Design of the small agricultural machinery Based on reconfigurable Module Method". Transactions of the Chinese society for agricultural machinery, Vol.36(4),pp.78-81. 